\title{
Beneficial Effect of a Thermal Spring Water on the Skin Barrier Recovery after Injury: Evidence for Claudin-6 Expression in Human Skin
}

\author{
Francine Joly $^{1}$, Cécile Gardille ${ }^{1}$, Eric Barbieux ${ }^{1}$, Luc Lefeuvre ${ }^{2}$ \\ ${ }^{1}$ Laboratoire SEPhRAPHARMA, Puteaux, France; ${ }^{2}$ Laboratoires Dermatologiques d'Uriage, Siège Social, Courbevoie, France. \\ Email: francine-joly@sephrapharma.com
}

Received September $3^{\text {rd }}, 2012$; revised October $5^{\text {th }}, 2012$; accepted October $14^{\text {th }}, 2012$

\begin{abstract}
Aim: To evaluate the possible advantages of dermo-cosmetic products formulated with thermal spring water instead of de-ionized water. We studied the effect of a dermo-cosmetic product on skin barrier recovery after injury. In one test the product was formulated with de-ionized water and again using the identical product formulated with thermal spring water. Study Design/Methods: Stratum corneum of human skin explants was removed (and not removed for the control) with an ether/acetone mixture. A cosmetic product, either formulated with de-ionized or thermal spring water, was then topically applied on the explants. After a 5-day incubation period, epidermal expression of aquaporine-3, filaggrin, claudin- 4 and claudin- 6 was studied by using immunohistochemical techniques. Results: The formulation containing de-ionized water seemed to act on the epidermal expression of filaggrin and claudin- 4 . When thermal spring water was used to make the formulation, we noted that this expression was greatly enhanced. The formulation containing thermal spring water also acted to increase aquaporin-3 and claudin- 6 expression. Conclusion: The use of thermal spring water in cosmetic formulations would appear to contribute to the cosmetic product's efficacy. It was also interesting to note that this study revealed claudin-6 expression in human skin for the first time.
\end{abstract}

Keywords: Keratinocyte Differentiation; Thermal Spring Water; Barrier Repair; Claudin-4; Claudin-6; Aquaporine 3; Filaggrin

\section{Introduction}

The stratum corneum is the outermost skin layer that protects an organism against exsiccation and infection (for a review, see [1]). When this epidermal layer is injured, the barrier function of the skin no longer functions. Stratum corneum injury can often occur after climatic, mechanical or chemical stress, after which it becomes essential to help skin to recover. Dermo-cosmetic actives have yet demonstrated their efficacy for this purpose (for a review, see [2]). In a stripped skin mice model, Lim et al. in 2007 [3] demonstrated that a combination of ursolic and oleanolic acids is able to increase expression of proteins which are important for skin moisturization (i.e. involucrin, loricrin and filaggrin). In 2003, Hara \& Verkman showed that defects observed in mice deficient in the epidermal water/glycerol transporter aquaporin-3 (AQP3)-i.e. reduced stratum corneum (SC) hydration and skin elasticity, and impaired barrier recovery after SC removalcould be corrected by systemic or topical administration of glycerol [4]. This is a humectants currently used incosmetics. In a complementary way, Dumas et al. show- ed in 2007 that a plant-extracted hydrating agent was able to significantly increase the keratinocyte AQP3 expression in a human reconstructed epidermis model [5]. Concerning the tight junction-related proteins which are greatly implicated in skin hydration and epidermal barrier function, we can also cite the work of Yahagi et al. who showed in 2011 that lysophospholipids (currently used in cosmetics) could improve skin moisturization by increasing the keratinocyte expression of occluding and claudin-1 [6].

In this study, we chose to determine whether the type of water used in formulations has a positive bearing on product efficacy. It is often said that thermal spring water is no more effective than de-ionized water. We formulated an anti-dry skin cosmetic product, with de-ionized or thermal spring water, and compared the effect of the two formulas on the expression of proteins implicated in the skin barrier function recovery in a human skin explants model. Furthermore, we decided to check for the presence in the human epidermis of a claudin recently shown to be essential for the murine epidermis differentiation and skin barrier function $[7,8]$, the claudin- 6 . 


\section{Materials and Methods}

\subsection{Reagents and Materials}

A surgical resection piece taken from a 34 year old Caucasian woman was used to make skin explants (diameter: $8 \mathrm{~mm}$ ). DMEM, Fetal calf serum and antibiotics were purchased from Life Technologies (Saint Aubin, France). Ether and acetone came from VWR International (Fontenay-sous-Bois, France). Mouse anti-human filaggrin, rabbit anti-human aquaporin-3, goat anti-human claudin4 and goat anti-human claudin- 6 primary antibodies came from Santa Cruz Biotechnologies (Santa Cruz, CA., USA). Goat anti-mouse secondary antibody coupled to Alexa Fluor ${ }^{\circledR} 488$, donkey anti-rabbit secondary antibody coupled to Alexa Fluor ${ }^{\circledR} 546$ and donkey anti-goat secondary antibody coupled to Alexa Fluor ${ }^{\circledR} 488$, were purchased from Life Technologies (Saint Aubin, France). Tissue-Tek $^{\circledR}$ came from VWR International (Leuven, Belgium). Dapi came from Roche Diagnostic (Mannheim, Germany). Thermal water came from Uriage-les-Bains, France.

Uriage thermal water: Ionic composition and osmolarity

Mean ionic content per liter:

$\begin{array}{lll}\text { Sulfates } & 2860 \mathrm{mg} \text { Potassium } & 45.5 \mathrm{mg} \\ \text { Chlorures } & 3500 \mathrm{mg} \text { Silicon } & 42 \mathrm{mg} \\ \text { Sodium } & 2360 \mathrm{mg} \text { Zinc } & 160 \mu \mathrm{g} \\ \text { Bicarbonates } & 390 \mathrm{mg} \text { Manganese } & 154 \mu \mathrm{g} \\ \text { Calcium } & 600 \mathrm{mg} \text { Copper } & 75 \mu \mathrm{g} \\ \text { Magnesium } & 125 \mathrm{mg} \text { Iron } & 15 \mu \mathrm{g} \\ \text { Osmolarity of Uriage thermal water is } 275 \pm 40 \\ \text { Osm. } \\ \text { Composition of the cosmetic formulations used } \\ \text { Product formulated with de-ionized water }\end{array}$

Aqua (water), cetearyl isononanoate, cetearyl ethylhexanoate, glycerine, polymethyl methacrylate, dimethicone, PFTE, acrylates/C10-30 alkyl acrylate crosspolymer, phenoxyethanol, cetyl alcohol, steareth-2, steareth21 , polyacrylate-13, perfume, sodium hydroxide, polyisobutene, benzoic acid, o-cymen-5-ol, biosaccharide gum14 , taurine, polysorbate 20 .

Product formulated with the Uriage thermal spring water

Aqua (water), Uriage thermal spring water, cetearyl isononanoate, cetearyl ethylhexanoate, glycerine, polymethyl methacrylate, dimethicone, PFTE, acrylates/C1030 alkyl acrylate crosspolymer, phenoxyethanol, cetyl alcohol, steareth-2, steareth-21, polyacrylate-13, perfume, sodium hydroxide, polyisobutene, benzoic acid, o-cymen-5-ol, biosaccharide gum-14, taurine, polysorbate 20 .

\subsection{Skin Explants Cultures and Treatments}

Normal human skin explants were cultured in Dubbelco modified Eagle medium (DMEM) with fetal calf serum $(10 \%)$ and antibiotics, in a humidified incubator under a $5 \% \mathrm{CO}_{2}, 95 \%$ air atmosphere.

For the study of filaggrin, aquaporin-3, claudin-4 and claudin-6 expression, stratum corneum of human skin explants were removed (and not removed in the control study) with an ether/acetone (1:1) mixture. A cosmetic product, either formulated with de-ionized or Uriage thermal spring water, was then topically applied on the explants. After a 5 day incubation period, epidermal expression of aquaporine-3, filaggrin, claudin-4 and claudin-6 was studied using immunohistochemical techniques.

\subsection{Filaggrin, AQP-3, Claudin-4 and Claudin-6 Expression Study}

At the end of the incubation period, skin explants were embedded in Tissue-Tek. Frozen Skin slices $(10 \mu \mathrm{m})$ were then realized by using a cryostat and fixed with acetone for a $10 \mathrm{~min}$ period. Non-specific binding sites were saturated by using fetal calf serum at $0.1 \%(\mathrm{v} / \mathrm{v})$ and the skin slices were incubated for 1 hour at room temperature with primary filaggrin, AQP-3, Claudin-4 and Claudin- 6 antibodies. The skin slices were rinsed with a phosphate buffered solution and incubated for 1 hour at room temperature in the dark with secondary antibodies coupled to fluorochromes (Alexa Fluor ${ }^{\circledR} 488$ for filaggrin, claudin-4 and claudin-6; Alexa Fluor ${ }^{\circledR} 546$ for AQP-3).

At the same time, cell nuclei were labeled with DAPI following the procedure described below: DAPI (dilution: $1 / 100)$ was added to the secondary antibodies in a solution containing $1 \%(\mathrm{w} / \mathrm{v})$ of bovine serum albumin.

\subsection{Immunohistochemical Images Acquisition}

The skin slices were observed with a fluorescence optical microscope. Appropriate filters were used to reveal Alexa Fluor ${ }^{\circledR} 488$, Alexa Fluor ${ }^{\circledR} 546$ and DAPI labeling, and images were obtained by using the dedicated software Archimed (Microvision Instruments, Evry, France).

\section{Results, Discussion and Conclusion}

As shown in Figure 1, in explants cultured in the control conditions (culture medium alone), filaggrin labeling revealed an expression of this protein in all the upper layers of the epidermis (A). When ether/acetone was applied (B), the stratum corneum was greatly altered and the filaggrin labeling became discontinuous. When explants were treated by the cosmetic product formulated with de-ionized water (C) after the delipidation procedure, a slight effect on the stratum corneum reparation seemed to be obtained. However, the filaggrin labeling 

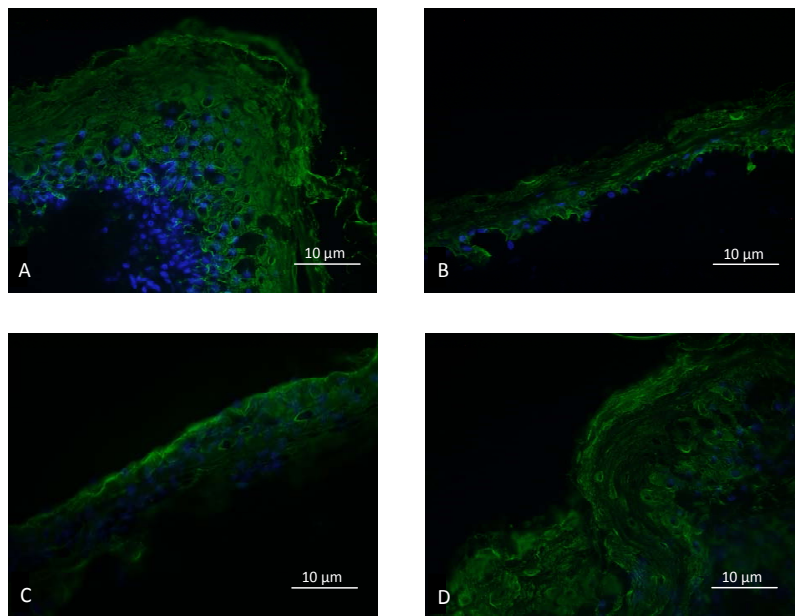

Figure 1. Epidermal filaggrin expression; A: Control. B: Delipidated explant. C: Delipidated explants treated with the cosmetic product formulated with de-ionized water. D: Delipidated explants treated with the cosmetic product formulated with thermal spring water.

remained poor and discontinuous. When explants were treated by the cosmetic product formulated with the Uriage thermal spring water (D), a very good effect on the stratum corneum reparation was observed and the filaggrin labeling was equivalent to the one obtained in the control conditions.

As shown in Figures 2(A)-(D), similar results were obtained for the AQP-3 expression study. The cosmetic product formulated with the Uriage thermal spring water was efficient in repairing the stratum corneum and in giving rise to an $\mathrm{AQP}-3$ labelling equivalent to the one observed in the control explants. Under the same experimental conditions, the product formulated with deionized water, had only a weak effect on the stratum corneum recovery and the AQP-3 expression.

As shown in Figures 3(A)-(D) and 4(A)-(D), the product formulated with the Uriage thermal spring water was also able to enhance the expression of the claudin- 4 and claudin- 6 in the delipidated explants. Again, the effect obtained with the product formulated with de-ionized water on the stratum corneum and the claudins expression, was significantly lower.

Taken together, these results emphasize the significance of formulating cosmetic products with thermal spring water instead of de-ionized water. Of course, the effectiveness of the tested products formulated with thermal water could be attributed to the ionic moiety of this type of water; calcium, magnesium and other ions have yet been described to significantly act on the epidermis differentiation and the formation and maintenance of the skin barrier function [9-12]. Nevertheless, the unique combination of ionic species existing in the Uriage thermal spring water could be of a great impor-
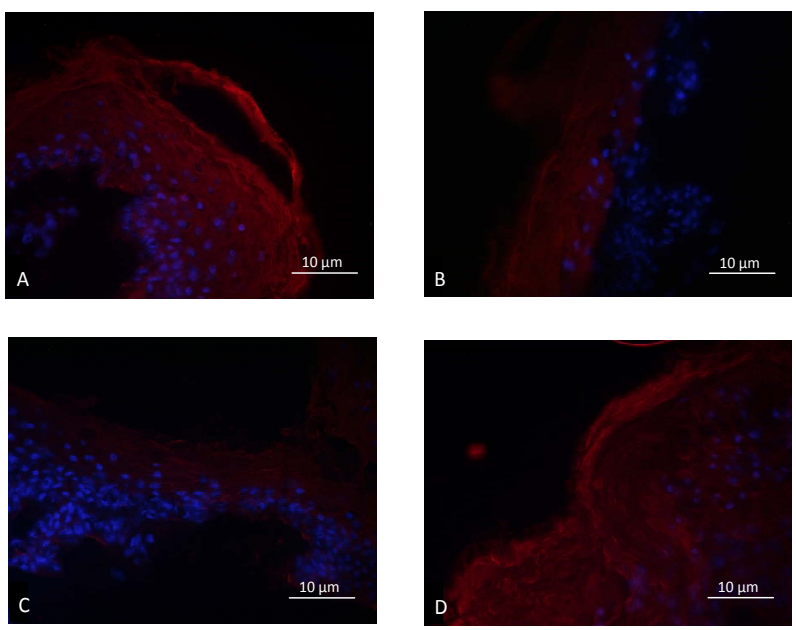

Figure 2. Epidermal aquaporin-3 expression; A: Control. B: Delipidated explant. C: Delipidated explants treated with the cosmetic product formulated with de-ionized water. D: Delipidated explants treated with the cosmetic product formulated with thermal spring water.
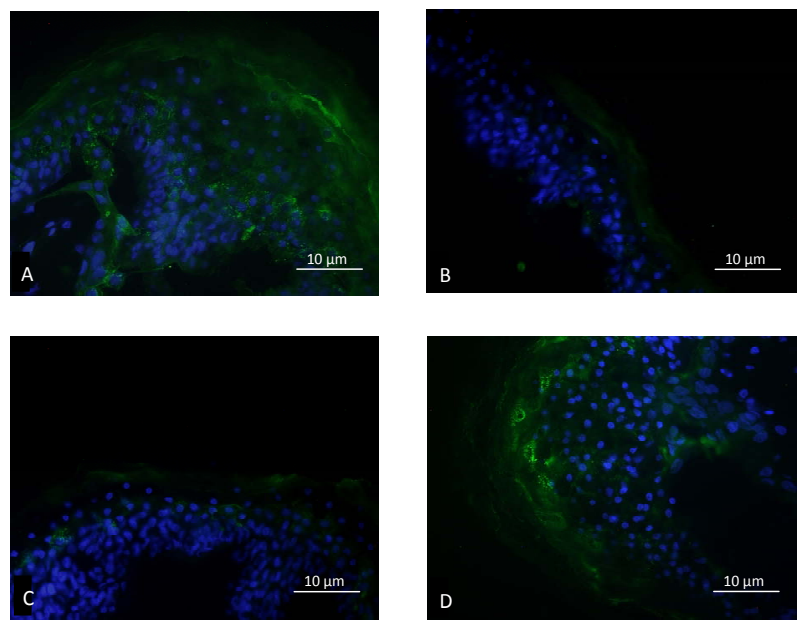

Figure 3. Epidermal claudin-4 expression. A: Control. B: Delipidated explant. C: Delipidated explants treated with the cosmetic product formulated with de-ionized water. D: Delipidated explants treated with the cosmetic product formulated with thermal spring water.

tance if we consider the possible interactions between these different types of ion. This hypothesis could be supported by the work of Collober et al. who showed in 1996 that the effect of Vittel water on the keratinocytes proliferation and differentiation was not linked to the sole presence of calcium, since the effect of a solution containing only calcium at the same concentration was lower [13]. Moreover, it is clear that the use of de-ionized water completed with some ionic species in defined conditions to formulate cosmetic products, seems too complicated and time-consuming to constitute an efficient strategy. In conclusion, the use of thermal spring 

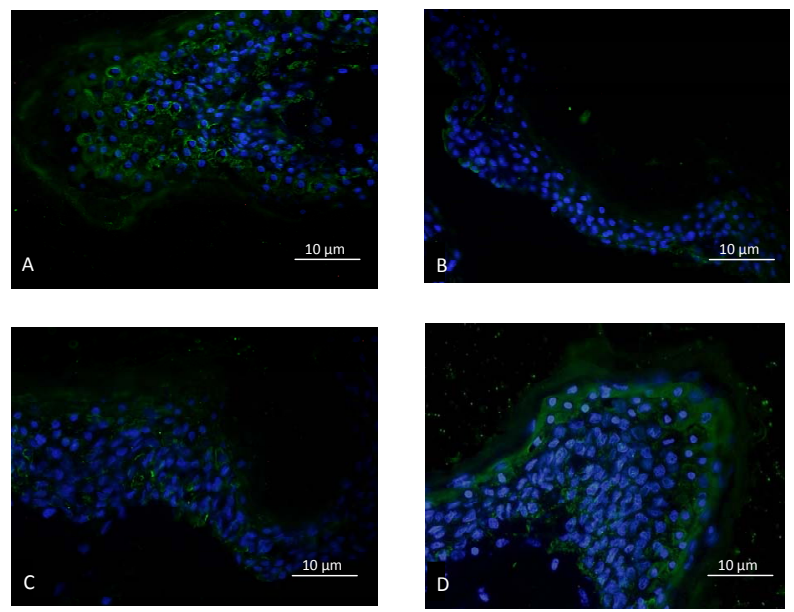

Figure 4. Epidermal claudin-6 expression; A: Control. B: Delipidated explant. C: Delipidated explants treated with the cosmetic product formulated with de-ionized water. D: Delipidated explants treated with the cosmetic product formulated with thermal spring water.

water in cosmetic formulation constitutes a benefit, not only for marketing considerations but also for efficacy ones.

In an innovative and interesting way, our study represents the first demonstration of the presence of claudin- 6 in human epidermis. Until now, this protein of the tight junction which is strongly implicated in formation and maintenance of the skin barrier function [8,9] had previously only been revealed in mice skin. As in the animal models, claudin-6 was shown to be implicated in dermatitis and age-related skin barrier dysfunctions; our study provides proof that a new target could be envisaged for physiopathologic considerations in human skin.

\section{Acknowledgements}

The authors are very thankful to $C$. Gardille (SEPhRAPHARMA) for her precious technical contribution.

\section{REFERENCES}

[1] A. Rawlings, "Recent Advances in Skin 'Barrier' Research," Journal of Pharmacy and Pharmacology, Vol. 62, No. 6, 2010, pp. 671-677.

[2] Z. Draelos, "Concepts in Skin Care Maintenance," Cutis, Vol. 76, No. 6S, 2005, pp. 19-25.

[3] S. Lim, et al., "Simultaneous Effect of Ursolic Acid and Oleanolic Acid on Epidermal Permeability Barrier Function and Epidermal Keratinocyte Differentiation via Peroxisome Proliferator-Activated Receptor-Alpha," The Jour- nal of Dermatology, Vol. 34, No. 9, 2007, pp. 625-634. doi:10.1111/j.1346-8138.2007.00344.x

[4] M. Hara and A. Verkman, "Glycerol Replacement Corrects Defective Skin Hydration, Elasticity, and Barrier Function in Aquaporin-3-Deficient Mice," Proceedings of the National Academy Science of the USA, Vol. 100, No. 12, 2003, pp. 7360-7365. doi:10.1073/pnas. 1230416100

[5] M. Dumas, et al., "Hydrating Skin by Stimulating Biosynthesis of Aquaporins," Journal of Drugs in Dermatology, Vol. 6, No. 6S, 2007, pp. 20-24.

[6] S. Yahagi, M. Koike, Y. Okano and H. Masaki, "Lysophospholipids Improve Skin Moisturization by Modulating of Calcium-Dependent Cell Differentiation Pathway," International Journal of Cosmetic Science, Vol. 33, No. 3, 2011, pp. 251-256. doi:10.1111/j.1468-2494.2010.00625.x

[7] T. Yuki, et al., "Characterization of Tight Junctions and Their Disruption by UVB in Human Epidermis and Cultured Keratinocytes," Journal of Investigative Dermatology, Vol. 131, No. 3, 2011, pp. 744-752. doi:10.1038/jid.2010.385

[8] A. Arabzadeh, T. Troy and K. Turksen, "Role of the Cldn6 Cytoplasmic Tail Domain in Membrane Targeting and Epidermal Differentiation in Vivo," Molecular and Cellular Biology, Vol. 26, No. 15, 2006, pp. 5876-5887. doi:10.1128/MCB.02342-05

[9] T. Troy, A. Arabzadeh, N. Lariviere, A. Enikanolaive and K. Turksen, "Dermatitis and Aging-Related Barrier Dysfunction in Transgenic Mice Overexpressing an Epidermal-Targeted Claudin-6 Tail Deletion Mutant," Plos One, Vol. 4, No. 11, 2009, p. e7814. doi:10.1371/journal.pone.0007814

[10] E. Proksch, J. Brandner and J. Jensen, "The Skin: An Indispensable Barrier," Experimental Dermatology, Vol. 17, No. 12, 2008, pp. 1063-1072. doi:10.1111/j.1600-0625.2008.00786.x

[11] P. Budtz, "Is Endogenous $\mathrm{Ca}^{++}, \mathrm{Mg}^{++}$-Dependent Endonuclease Activity Involved in Epidermal Terminal Differentiation?" Journal of Investigative Dermatology, Vol. 98, No. 6, 1992, p. 945. doi:10.1111/1523-1747.ep12461065

[12] I. Tenaud, I. Sainte-Marie, O. Jumbou, P. Litoux and B. Derno, "In Vitro Modulation of Keratinocyte Wound Healing Integrins by Zinc, Copper and Manganese," British Journal of Dermatology, Vol. 140, No. 1, 1999, pp. 26-34. doi:10.1046/j.1365-2133.1999.02603.x

[13] I. Collober, M. Noel-Hudson, J. Wepierre and C. Montastier, "Activity of Vittel Water on Proliferation of $\mathrm{Hu}-$ man Fibroblasts, Proliferation and Differentiation of $\mathrm{Hu}$ man Keratinocytes," International Journal of Cosmetic Science, Vol. 16, No. 4, 1994, pp. 149-160. doi:10.1111/j.1467-2494.1994.tb00093.x 